Brit. J. industr. Med., 1951, 8, 26.

\title{
NEW LIQUID SKIN CLEANSERS FOR WORKERS EXPOSED TO MINERAL OIL
}

\author{
BY \\ L. N. SAVIDGE and F. H. TYRER \\ From the Research Department, Port Sunlight
}

(RECFIVED FOR PUBLICATION SEPTEMBER 14, 1950)

The importance of providing adequate cleansing facilities for industrial workers, and in particular for those whose skin is constantly or frequently exposed to contamination with potentially harmful lubricating oils, is now well recognized. The removal of lubricating oils from the skin, although superficially an apparently simple matter, is, in fact, usually difficult to accomplish without using harsh abrasive cleansers or oil-solvents such as kerosene or white spirit. Traces of lubricating oils are tenaciously retained, particularly in the hair follicles, and are a potential source of danger. Whilst abrasive and solvent cleansers are very popular with workmen because of the ease and speed with which they can be used, both must be condemned, the one because of the damage to the epidermis resulting from frequent use, and the other because of its excessive defatting action, which may lead to dermatitis. In considering the ideal type of cleanser, therefore, due account must be paid to its effectiveness in the removal of oily and particulate soils, to the absence of abrasion or excessive defatting effect, to ease of application, and speed of action. The last two factors are important if the worker is to be persuaded to use the cleanser. It follows then that cleansers supplied to the workers must be chosen carefully, and in making this choice it is desirable that the industrial medical officer should be consulted.

Fortunately the assessment of the efficiency of a cleanser is a simple matter, as has been shown by Cruickshank (1948). In order to see whether any lubricating oil is left on the skin after washing, it is sufficient to examine the hands and arms of the worker in ultra-violet light, when the presence of the merest residual traces of such oil will be disclosed by a bright, pale blue fluorescence. Using a special washing technique and then applying this test for residual lubricating oil, Cruickshank compared a wide variety of cleansers and found the most effective to be a mixture of

$$
\begin{aligned}
& \text { Powdered soap * .. } \quad . . \quad \ldots \quad \ldots \quad \ldots \quad 50 \% \\
& \text { Mild organic absorbent such as wood } \\
& \begin{array}{llllll}
\text { flour .. } & . . & . . & . & . & 42 \%
\end{array}
\end{aligned}
$$

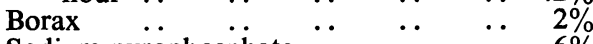

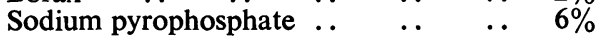

We have found, however, in extensive trials, that the regular use of this cleanser tends to be rather drying to the skin of some individuals, pariicularly in cold weather and with elderly workers, and that this can be rectified by omission of the pyrophosphate without any noticeable loss of efficiency in removing dirt and oil. Cruickshank found the next most effective cleansers to be those based on soap or sulphonated oils in conjunction with white spirit or a mixture of white spirit and light lubricating oil together with minor ingredients and water. These liquid cleansers rapidly loosened the dirt and oil from the hands, but during rinsing some of the lubricating oil was re-deposited on the skin and in ultra-violet light could be seen as a fine, fluorescent film; a second wash, preferably with soap and water, was found to be necessary in order to remove the oil completely from the skin. We carried out wide scale industrial trials under ordinary conditions of use and confirmed these findings.

\section{New Type Liquid Cleansers}

In discussing alternative methods of skin cleansing Cruickshank found that complete removal of lubricating oil could be obtained if the hands and arms were anointed with medicinal paraffin or other bland oil, and then washed. Thus a cleanser containing medicinal paraffin or a similar innocuous oily constituent might prove to be the most effective type of liquid cleanser. We have examined a

* Powdered soap is obtained by drying soap to which no alkali or other salts have been added. 
number of such cleansers, which incidentally are far more difficult to formulate than those based on kerosene or white spirit, and have found them to be highly efficient although perhaps not quite so effective as the wood flour cleanser mentioned above when heavy contaminations of particulate soil have to be removed. An example of this type of cleanser is a clear, single phase liquid containing the following :

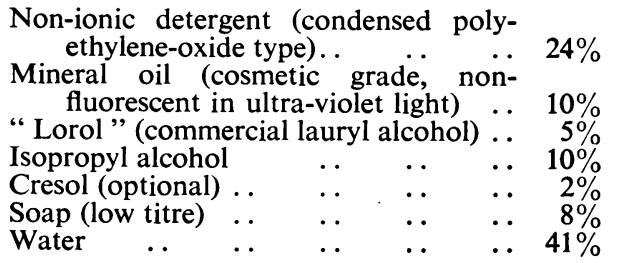

This cleanser, rubbed into the dry skin, loosens and dissolves the contaminating lubricating oil and when rinsed off with water leaves the skin free from the soil. In exceptionally difficult cases a second wash may be necessary, and the cleanser may be used on the already wetted skin. A further virtue of this particular type of cleanser is that the skin is left particularly soft and smooth and less liable to chap in cold weather.

A cleanser such as the one just described is necessarily rather expensive to produce and on this account might not be generally acceptable, although limited experience suggests that the expense is justified in cases where the worker is sensitive to the usual cleansers. Synthetic detergents or soap used alone, as Cruickshank showed, are not very satisfactory cleansers for oily dirt, although, in the case of certain of the synthetics, lack of efficiency is due to difficulties of application rather than to lack of detergent properties. It has now been found that suitable mixtures of synthetic detergents and soap form cleansers both effective and pleasant to use. A cleanser containing

\begin{tabular}{|c|c|c|c|c|}
\hline \multicolumn{5}{|c|}{ Non-ionic detergent (condensed poly- } \\
\hline ethylene-oxid & pe) & & & \\
\hline p (low titre) & $\ldots$ & & & \\
\hline propyl alcohol & $\because$ & & & \\
\hline ter $\ldots$ & $\ldots$ & . & . & $76.5^{\circ}$ \\
\hline
\end{tabular}

when used on the dry skin as previously described, is effective in removing most oily soils, and is very little inferior to the compound cleanser containing cosmetic grade mineral oil. This cleanser has been tested under ordinary conditions of use by large

\section{Photographs in ultra-violet light.}

FIG. 1.-Normal hand before soiling, with natural fluorescence of nails.

FIG 2.-Hand after soiling with dirty diesel engine oil.

FIG. 3.-Same hand after washing with toilet soap.

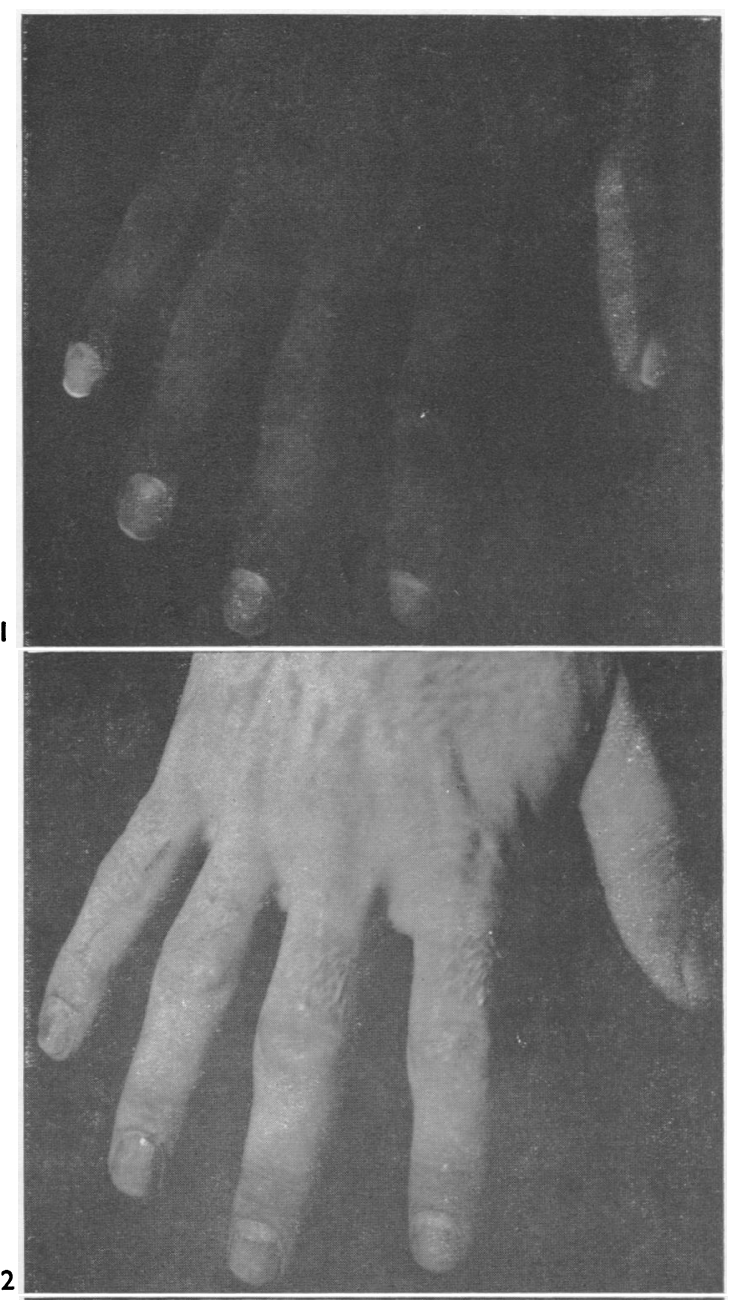

2

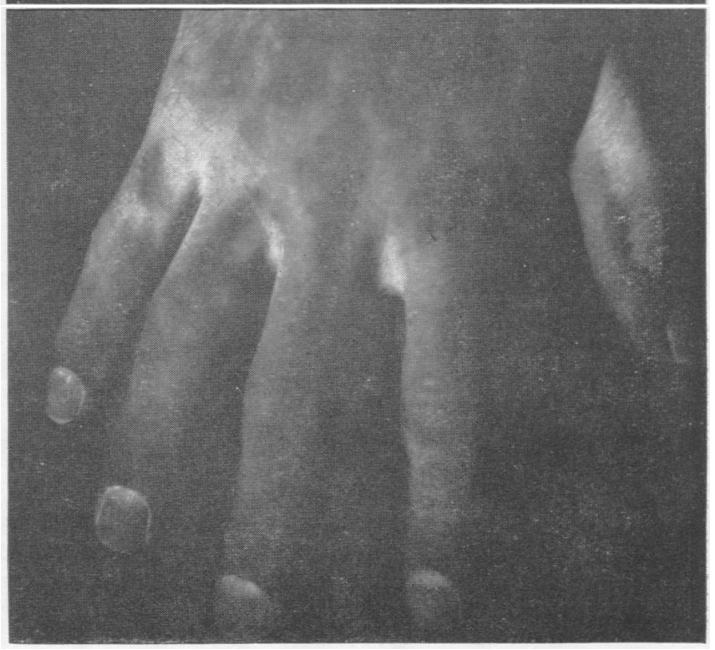




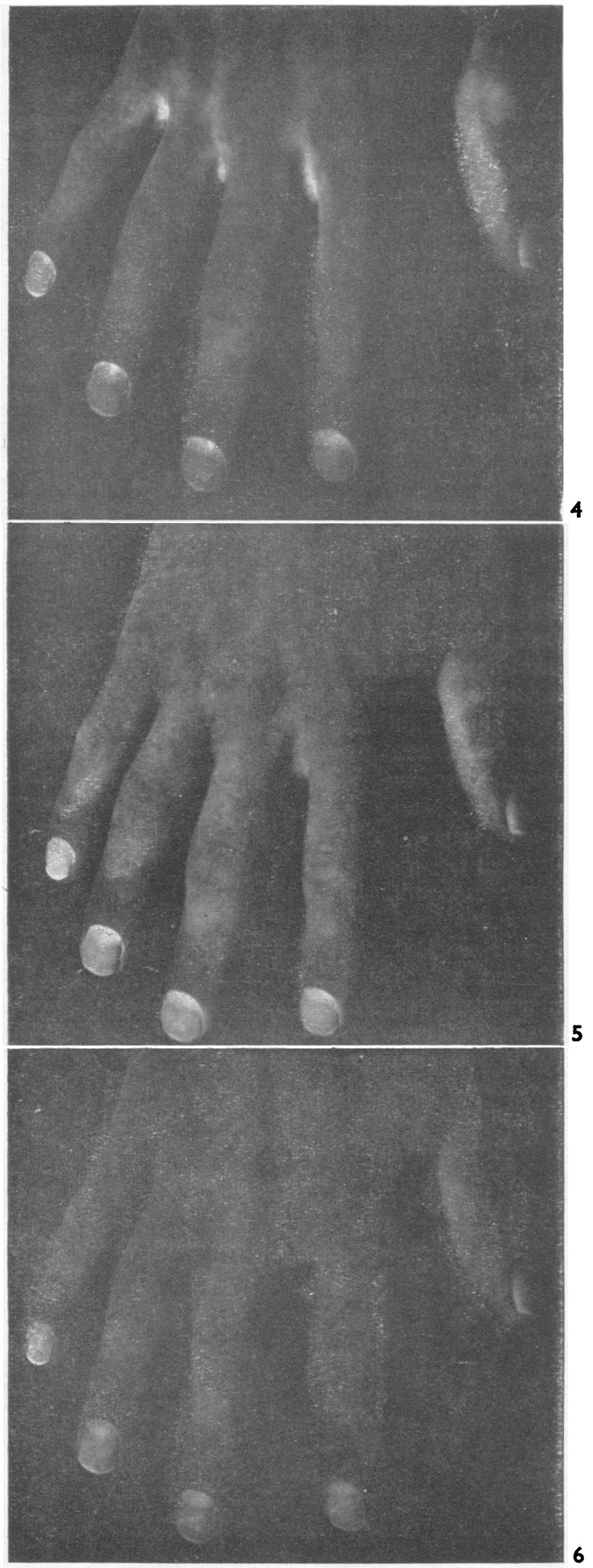

numbers of workers such as fitters, garage hands, and others whose work involves the handling of dirty lubricating oils and greases, and by women handling animal and vegetable oils, with whom it proved very popular as it left the hands clean, soft, and smooth.

\section{Efficiency of Different Types of Cleanser}

Some idea of the relative efficiencies of the various types of cleansers mentioned may be obtained from the photographs, which were taken in ultra-violet light through a gelatine screen to filter out stray ultra-violet radiations. Fig. 1 shows the normal hand before soiling; apart from the finger nails, which fluoresce naturally, the hand is quite free from fluorescence. Fig. 2 shows the appearance of the hand after soiling with dirty sump oil from a diesel engine; the whole hand shines with a bright, pale blue fluorescence. Fig. 3 shows the same hand after washing with a good toilet soap ; it will be seen that considerable areas of the skin are still contaminated with oil. The hand washed with a proprietary compound cleanser containing turkey red oil, pine oil, and kerosene (Fig. 4) is seen to retain oil, particularly between the fingers and at the wrist, and not to be entirely free from contamination elsewhere. Figs. 5 and 6 show the hands after washing with the wood flour/soap cleanser and the liquid cleanser based on the mixture of soap and non-ionic detergent, respectively; both hands are quite free from fluorescent oil.

\section{Summary}

The most effective hand cleanser is a powder containing about $50 \%$ wood flour, $48 \%$ powdered 5 soap, and $2 \%$ borax. For those who prefer a liquid cleanser, either because of ease of dispensing, feel, or for any other reason, a single phase liquid containing a bland, non-fluorescent mineral oil, a non-ionic detergent and soap, together with blending agents, can strongly be recommended, particularly for use where the skin is sensitive to other cleansers. An almost equally effective liquid cleanser of a less expensive type is one based on a mixture of non-ionic detergent and soap.

\section{REFERENCE}

Cruickshank, C. N. D. (1948). British Journal of Industrial Medicine, 5, 204.

Fig. 4.-After washing with proprietary cleanser.

FIG. 5.-After washing with wood flour/soap cleanser.

Fig. 6.-After washing with non-ionic detergent/soap cleanser. 